\title{
Psychosocial Factors Associated with Transfer Anxiety among Open Heart Surgery Patients Transferred from the Intensive Care Unit to the General Ward
}

\author{
Jaya Rijal ${ }^{\circledR}$, Wipa Sae-Sia*, Luppana Kitrungrote \\ Department of Adult and Elderly Nursing, Faculty of Nursing, Prince of Songkla University, Hat Yai, Thailand \\ Email: *wipa.sa@psu.ac.th
}

How to cite this paper: Rijal, J., Sae-Sia, W., and Kitrungrote, L. (2020) Psychosocial Factors Associated with Transfer Anxiety among Open Heart Surgery Patients Transferred from the Intensive Care Unit to the General Ward. Health, 12, 1583-1597. https://doi.org/10.4236/health.2020.1212115

Received: November 17, 2020

Accepted: December 18, 2020

Published: December 21, 2020

Copyright $\odot 2020$ by author(s) and Scientific Research Publishing Inc. This work is licensed under the Creative Commons Attribution International License (CC BY 4.0).

http://creativecommons.org/licenses/by/4.0/

\section{(c) (i) Open Access}

\begin{abstract}
Introduction: Patient's transfer from the intensive care unit (ICU) to the general ward indicates their improving health status. However, the transfer produces anxiety when patients enter an unfamiliar environment with different care protocols and circumstances. Objectives: This study aimed to examine the level of ICU transfer anxiety among open heart surgery patients and determine the psychosocial factors associated with ICU transfer anxiety among open heart surgery patients. Methods: Data were collected in a cardiac center in Kathmandu City, Nepal among 95 open heart surgery patients within 24 hours of their transfer from an ICU to a general ward. The study used four self-reported questionnaires, namely the modified Mishel Uncertainty in Illness Scale for Adults, modified Brief COPE Inventory, Nurses' Support Questionnaire, and State Anxiety Inventory. Results: Fifty-two patients $(54.7 \%)$ had a high level of transfer anxiety. Spearman's rank correlation showed that uncertainty in illness, coping, and nurses' support were significantly related to transfer anxiety $(p<0.001)$. Conclusion: The results of this study suggest nurses to address uncertainty in illness of the patients, improve their coping abilities, and provide need-based nursing support to them during the transitional phase. Besides, clinicians and governmental agencies should contribute to implication of transitional guidelines, which can reduce transfer anxiety and promote health and recovery of the patients.
\end{abstract}

\section{Keywords}

Transfer Anxiety, Uncertainty, Coping, Intensive Care Unit, Open Heart Surgery 


\section{Introduction}

Transition of patients from an intensive care unit (ICU) to a general ward is an indicator of the patient's recovery that comes with mixed feelings. Patients were delighted to meet their relatives in the general ward and spend time with them [1]. However, transfer is associated with patients' stress and anxiety [2] because of their separation from monitoring devices and staff personnel in the ICU [1]. Patients need to adjust to an unfamiliar ward environment with different care protocols and new faces of healthcare staff [1] [3]. It is a known fact that the ICU has more resources available including advanced technological appliances, specialized healthcare professionals, and a timely multidisciplinary team approach compared to a general ward [4]. Therefore, patients perceived ward circumstances as unsafe and insecure [1] and felt uncertain about getting their needs promptly addressed [5].

North American Nursing Diagnosis Association defined transfer anxiety as the state of emotion that produces physical and mental disturbances on transfer from one place to another [6]. Two studies done in the Middle East region of the world reported that $63 \%$ of patients transferred from the ICU to the general ward suffered from transfer anxiety [7] and $63.6 \%$ of post-cardiac surgical patients had moderate transfer anxiety [8].

Discharge from the cardiovascular surgical ICU to a step-down unit can produce a negative physio-psychological effect on a patient's health [9]. They developed symptoms, such as pain, fatigue, anorexia, sleep imbalance, and felt powerless, helpless, and abandoned [3] [5] [10]. In addition, patients encountered issues with memory and orientation including hallucination and delusion in the early days of transfer to a general ward [4] [10]. These adverse events can result in ICU readmission and a lengthened hospital stay [11].

According to a stress and coping model proposed by Lazarus and Folkman [12], anxiety is a negative emotional health outcome when a person feels uncertain, uses ineffective coping, and perceives limited social support. Previous studies found a relationship between uncertainty, coping, and the support system and anxiety. Studies mentioned that patients were uncertain about the appropriateness of their discharge from the ICU to the ward and the symptoms that would persist after the transfer, which escalated transfer anxiety [3] [5]. In addition, problem-focused coping was negatively correlated with anxiety among open heart surgery patients [13]. Moreover, anxiety was reported to disturb adaptive coping abilities of the patients [14]. Similarly, studies determined that the nurses' support towards physical, psychological, and emotional dimensions in patients were effective in minimizing ICU transfer anxiety [15] [16].

The aforementioned studies related to factors associated with transfer anxiety were mostly represented by the Middle East and Western countries. However, religion, beliefs, social relationships, and the care environment, such as hospital care services, of those countries differ from Nepal, which may influence the coping, social resource, and health outcomes of Nepalese patients. Therefore, it 
is necessary to examine transfer anxiety and its psychosocial factors among open heart surgery patients such that nurses can be aware and consider the factors while implementing interventions to reduce transfer anxiety.

The objectives of this study were: 1) to examine the level of ICU transfer anxiety among open heart surgery patients, and 2) to determine the psychosocial factors associated with ICU transfer anxiety among open heart surgery patients.

\section{Methods}

\subsection{Study Design and Setting}

This was a descriptive correlational cross-sectional survey carried out among open heart surgery patients transferred from the ICU to the general ward in a cardiac center in Nepal. The center has 10 ICU beds allocated for adult surgical patients with an additional 3-bed capacity. There are total 16 beds in the general ward. Usually, the nurse-patient ratio in the ICU is $1: 3$, whereas the nurse-patient ratio in the general ward is $1: 8$. The patients who are eligible for discharge to the general ward are accompanied by an ICU nurse and the available relatives of the patients during transfer.

\subsection{Ethical Approval}

This study was approved by the Social and Behavioral Sciences, Institutional Review Board, Prince of Songkla University, Thailand [2017-Nst-Qn 049] and the Nepal Health Research Council, Nepal [Reg. 501/2017]. Also, permission from the cardiac center [Ref. no. 10-2018] was obtained.

\subsection{Sample and Inclusion/Exclusion Criteria}

A sample size of 95 was calculated based on a power of 0.80 , significance level of 0.05 [17], and an estimated correlation coefficient of 0.27 [18]. All open heart surgery patients discharged from the ICU to the general ward in the study setting with the following inclusion criteria were included: 1) having first experience of open heart surgery; 2) hemodynamically stable; 3 ) 18 years and older, and 4) able to speak in Nepali language.

\subsection{Research Instruments}

Patient information and four self-reported questionnaires were used in collecting the data, namely the modified form of Mishel Uncertainty in Illness Scale for Adults (MUIS), the modified form of Brief COPE Inventory, Nurses' Support Questionnaire (NSQ), and State Anxiety Inventory (SAI).

Patient information consisted of demographic and clinically relevant data including age, sex, educational status, employment status, monthly family income, surgical procedures, the length of ICU stay, and post-operative signs and symptoms which were obtained from patient interviews and the medical records.

The modified form of MUIS [19] was employed to measure uncertainty in illness. It is a 5-point Likert scale with 33 items ranging from "strongly disagree" 
(1) to "strongly agree" (5). The total possible score is between 33 and 165. A higher score represents higher perceived uncertainty in illness.

The modified form of the Brief COPE Inventory [20] determined the coping. Coping was segregated into 3 dimensions, namely emotion-focused coping, problem-focused coping, and dysfunctional coping [21]. The 4-point Likert scale had 26 items with scores of 1 (I have not been doing this at all) to 4 (I have been doing this a lot). The scores for emotion-focused coping (10 items), problem-focused coping (8 items), and dysfunctional coping (8 items) were (10 - 40), $(8-32)$, and (8 - 32), respectively. Higher scores in the dimensions indicate more use of that coping measure.

The NSQ, which was developed by the researchers based on a literature review of experimental studies [15] [16] investigated nurses' support. It consisted of 19 items on a 5 -point Likert scale ranging from 1 (not at all) to 5 (to a greater extent). The total score could vary from 19 to 95 . A higher score denotes higher nurses' support.

The SAI [22] was used to assess transfer anxiety. It is a 20 -item 4-point Likert scale that ranged from "not at all" (1) to "very much so" (4). The sum scores could vary from 20 to 80 . High scores describe a higher presence of transfer anxiety. This study follows the SAI classification by Zakerimoghadam, Ghyasvandian, Asayesh and Jodaki [8] that divides the anxiety of post-cardiac surgery patients transferred from an ICU to a general ward into three levels as low (20 $39)$, moderate (40 - 59), and high (60 - 80).

\subsection{Validity and Reliability of the Research Instruments}

The modified MUIS, modified Brief COPE Inventory, NSQ, and SAI questionnaires were validated by a panel of five experts. A scale content validity index (S-CVI) greater than 0.80 [17] ensured the accuracy of the questionnaires. The S-CVI values were $0.99,0.95,0.96$, and 1.00 for the modified MUIS, modified Brief COPE Inventory, NSQ, and SAI, respectively. The back-translation technique by Brislin [17] was used to translate the questionnaires into Nepali language. The internal consistency reliability of the Nepali versions of the questionnaires in all 95 patients revealed that the Cronbach's alpha coefficient of the modified MUIS, NSQ, and SAI were $0.96,0.91$, and 0.97, respectively. In addition, the Cronbach's alpha coefficient of the Brief COPE Inventory for problem-focused coping, emotion-focused coping, and dysfunctional coping were $0.92,0.83$, and 0.60 , respectively.

\subsection{Data Collection}

The patients willing to enroll in the study were approached and requested to sign the written informed consent. After obtaining the consent, patients completed the four self-reported questionnaires when they felt able within 24 hours of getting transferred to the general ward. The survey was conducted between February and March 2018. 


\subsection{Statistical Analysis}

The data were analyzed by computer software SPSS version 16.0. Descriptive statistics were used to report the patient information, and the study variables. The distribution of transfer anxiety scores violated the assumption of normality. Hence, transfer anxiety has been reported in terms of median and interquartile range. Spearman's rank correlation was used to test the relationship between the study variables. The significance level was set as $p<0.05$.

\section{Results}

\subsection{Patient Information}

A total of 95 open heart surgery patients were enrolled in this study within 24 hours of their transfer from an ICU to a general ward. Table 1 displays the

Table 1. Demographic and clinically relevant data of patients $(N=95)$.

\begin{tabular}{|c|c|c|}
\hline Variables & $n(\%)$ & Mean $\pm S D$ \\
\hline Age (years) & & $44.15 \pm 13.75$ \\
\hline$\leq 40$ & $39(41.4)$ & \\
\hline$>40$ & $56(58.9)$ & \\
\hline \multicolumn{3}{|l|}{ Sex } \\
\hline Male & $42(44.2)$ & \\
\hline Female & $53(55.8)$ & \\
\hline \multicolumn{3}{|l|}{ Educational status } \\
\hline Uneducated & $31(32.6)$ & \\
\hline Formally educated & $64(67.4)$ & \\
\hline \multicolumn{3}{|l|}{ Employment status } \\
\hline Unemployed & $72(75.8)$ & \\
\hline Employed & $23(24.2)$ & \\
\hline Monthly family income (USD) & & $372.63 \pm 168.65$ \\
\hline$\leq 300$ & $48(50.5)$ & \\
\hline$>300$ & $47(49.5)$ & \\
\hline \multicolumn{3}{|l|}{ Surgical procedures } \\
\hline Congenital cardiac repair & $18(18.9)$ & \\
\hline Valvular heart surgery & $44(46.3)$ & \\
\hline Coronary artery bypass graft & $28(29.5)$ & \\
\hline Others & $5(5.3)$ & \\
\hline Length of ICU stay (days) & & $2.76 \pm 2.27$ \\
\hline$\leq 3$ & $81(85.3)$ & \\
\hline$>3$ & $14(14.7)$ & \\
\hline \multicolumn{3}{|c|}{ Presence of post-operative signs and symptoms } \\
\hline Dysrhythmia & $35(36.8)$ & \\
\hline Palpitation & $78(82.1)$ & \\
\hline Pain & $95(100.0)$ & \\
\hline Nausea & $70(73.7)$ & \\
\hline Anorexia & $84(88.4)$ & \\
\hline Insomnia & $42(44.2)$ & \\
\hline
\end{tabular}

Note: $S D=$ Standard Deviation, USD $=$ US Dollars, ICU = Intensive Care Unit. 
demographic and clinically relevant data of the patients. More than half of the patients were older than 40 years $(n=56,58.9 \%)$ and were females $(n=53$, $55.8 \%)$. Thirty-one uneducated patients (32.6\%) were not completely illiterate. The majority of the patients $(n=72,75.8 \%)$ were unemployed and most of them were engaged as home manager, farmer, or student. Valvular heart surgery was the most common operation done $(n=44,46.3 \%)$. The mean length of ICU stay was 2.76 days. All patients complained of pain and the majority of them had post-operative symptoms of anorexia $(n=84,88.4 \%)$, palpitation $(n=78$, $82.1 \%)$, and nausea $(n=70,73.7 \%)$.

\subsection{Transfer Anxiety and Psychosocial Factors}

Table 2 illustrates the descriptive data of transfer anxiety and the psychosocial factors. The median of $60(I Q R=21)$ indicated that open heart surgery patients in this study had a high level of anxiety on transfer from an ICU to a general ward, and high transfer anxiety was found among 52 patients (54.7\%) (Table 3). The interpretation of the average values of uncertainty in illness and problem-focused coping suggested moderate scores, whereas emotion-focused coping and nurses' support had high scores. Dysfunctional coping was the only variable that had a quiet low score. Therefore, it can be said that the patients in this study had moderate levels of uncertainty in illness and problem-focused coping, high levels of emotion-focused coping and nurses' support, and a low level of dysfunctional coping.

\subsection{Psychosocial Factors Associated with Transfer Anxiety}

The correlation matrix reported significant positive correlations between transfer

Table 2. Transfer anxiety and psychosocial factors of patients.

\begin{tabular}{cccc}
\hline Variables & Min-Max & Median $(I Q R)$ & Mean $\pm S D$ \\
\hline Transfer anxiety & $27-72$ & $60(21)$ & - \\
Uncertainty in illness & $60-121$ & - & $89.86 \pm 15.99$ \\
Emotion-focused coping & $16-31$ & - & $22.01 \pm 3.33$ \\
Problem-focused coping & $10-27$ & - & $17.94 \pm 4.50$ \\
Dysfunctional coping & $8-23$ & - & $13.67 \pm 3.11$ \\
Nurses' support & $59-88$ & - & $72.41 \pm 7.68$ \\
\hline
\end{tabular}

Note: $I Q R=$ Interquartile Range, $S D=$ Standard Deviation.

Table 3. Frequency and percentage of transfer anxiety in patients categorized by levels of transfer anxiety.

\begin{tabular}{cc}
\hline Level of transfer anxiety & $\boldsymbol{n}(\%)$ \\
\hline Low $(20-39)$ & $20(21.1)$ \\
Moderate $(40-59)$ & $23(24.2)$ \\
High $(60-80)$ & $52(54.7)$ \\
\hline
\end{tabular}


Table 4. Transfer anxiety and psychosocial factors of patients.

\begin{tabular}{lcccccc}
\hline \multicolumn{1}{c}{ Variables } & $\mathbf{1}$ & $\mathbf{2}$ & $\mathbf{3}$ & $\mathbf{4}$ & $\mathbf{5}$ & $\mathbf{6}$ \\
\hline 1. Transfer anxiety & 1 & & & & & \\
2. Uncertainty in illness & $0.90^{*}$ & 1 & & & & \\
3. Emotion-focused coping & $-0.60^{*}$ & $-0.66^{*}$ & 1 & & & \\
4. Problem-focused coping & $-0.80^{*}$ & $-0.86^{*}$ & $0.59^{*}$ & 1 & & \\
5. Dysfunctional coping & $0.72^{*}$ & $0.76^{*}$ & $-0.63^{*}$ & $-0.68^{\star}$ & 1 & \\
6. Nurses' support & $-0.83^{*}$ & $-0.89^{*}$ & $0.73^{*}$ & $0.88^{*}$ & $-0.73^{*}$ & 1 \\
\hline
\end{tabular}

Note: ${ }^{*} p<0.001$.

anxiety and uncertainty in illness $\left(r_{s}=0.90, p<0.001\right)$ and transfer anxiety and dysfunctional coping $\left(r_{s}=0.72, p<0.001\right)$ which denoted that patients with higher uncertainty in illness and dysfunctional coping had developed higher transfer anxiety. However, a significant negative correlation of transfer anxiety with emotion-focused coping $\left(r_{s}=-0.60, p<0.001\right)$, problem-focused coping $\left(r_{s}\right.$ $=-0.80, p<0.001)$, and nurses' support $\left(r_{s}=-0.83, p<0.001\right)$ indicated that the more emotion-focused coping and problem-focused coping patients used and the more nurses' support they obtained, the less transfer anxiety they perceived (Table 4).

\section{Discussion}

This study evaluated the level of ICU transfer anxiety and its association with the psychosocial factors. The high level of transfer anxiety was most prevalent among the patients and it was found that uncertainty in illness, patients' coping, and nurses' support were significantly correlated to transfer anxiety.

The majority of patients in this study had high transfer anxiety which contradicted a study in Iran where the frequency was greater in moderate transfer anxiety [8]. In the cardiac center in Nepal, a specific transitional care guideline is not practiced, and preparation of patient transfer depends on the experience of the individual nurse. Thus, failing to implement standardized care is the probable cause of high level of transfer anxiety [23].

Uncertainty established a highly positive correlation with transfer anxiety in the present study. Patients admitted to the ICU felt uncertain of the future events they should deal with in the general ward [23] [24]. Patients were unsure of being frequently monitored and getting their concerns addressed due to the high nurse-to-patient ratio in the general ward compared to ICU [24]. The findings of this study were similar to a systematic review which reported the coexistence of transfer anxiety with uncertainty or fear of the new environment and the lack of close observation [25].

A study by Tung, Hunter and Wei [13] done among cardiac surgery patients reported a significant negative association between problem-focused coping and anxiety. Although the previous study was not specific to transfer anxiety, the result was congruent to the current study. This may be explained by the fact that 
social support is an indirect source of adaptive coping [12]. For example, the family caregiver assisted and advised patients to deal with stressful situations of the general ward. Therefore, it strengthened problem-focused coping and reduced anxiety [26].

Similarly, faith in God and having a religious belief is a crucial part of Nepalese society and has been known to provide psychological comfort and develop positive thoughts [27]. The existing spiritual influences among patients might have shown a negative relationship between emotion-focused coping and transfer anxiety in the present study.

In addition, patients after cardiac surgery find it difficult to validate the response to emotions and end up adapting dysfunctional coping strategies such as denial [28]. Hence, patients in this study were possibly in the denial phase and believed that their condition was not real, which resulted in a positive correlation of dysfunctional coping and transfer anxiety.

The present study aligns with earlier studies which evidenced that increased nurses' support can reduce ICU transfer anxiety [15] [16]. However, the level of transfer anxiety appeared to be high because the support provided by the nurses may not rely on the patients' transitional problems [23]. A high number of patients in this study had symptoms, such as pain, palpitation, and anorexia at the general ward (Table 1). In addition, none of the patients were informed about dealing with those post-operative symptoms. Nurses' support was focused mostly on providing general information regarding the orientation and visiting hours of the general ward.

\section{Limitations}

This study has some limitations. First, the results of the study cannot be generalized to open heart surgery patients who were transferred from an ICU to a general ward where the context of patient care differs from that in Nepal. Furthermore, the reliability of the dysfunctional coping dimension of the Brief COPE Inventory is much lower than the other two dimensions; therefore, psychometric properties of the dysfunctional coping should be re-evaluated in the future. Also, the influence of these psychosocial factors on transfer anxiety can be further identified through a predictive study design.

\section{Recommendations for Clinical Practice}

This study showed the magnitude of transfer anxiety in open heart surgery patients in Nepal compared to Middle East and Western countries of the world. Since prior studies focused on the proper implication of transitional care guidelines or standard care to reduce ICU transfer anxiety, it is suggested that the health ministry be aware and formulate transfer protocol, incorporate it into the country's health policy, and conduct regular follow-ups of its implementation in the hospitals. Similarly, more nurses should be recruited to limit the negative consequences of high nurse-patient ratio on patient's quality care. 
Upon having an understanding of the effects of transfer anxiety on patients' recovery, nurses are advised to assess the level of transfer anxiety in patients and act accordingly. The results of this study exhibited strong relationships between uncertainty in illness, coping, nurses' support, and ICU transfer anxiety. Therefore, it is recommended that nurses be concerned with individual uncertainty and coping abilities of the patients. In order to manage transfer anxiety, nurses should enhance appropriate utilization of problem-focused and emotion-focused coping measures among patients. Moreover, nurses are suggested to provide patient-centered emotional and informational support during the transitional period.

\section{Conclusion}

Transfer from the ICU to the general ward is a major source of anxiety among patients in Nepal. This study reported uncertainty in illness, coping, and nurses' support as the psychosocial factors related to ICU transfer anxiety. Thus, these factors should be individually addressed to reduce transfer anxiety among patients. Collaborative efforts by the government, hospital administration, clinicians, and nurses are crucial in adapting appropriate transitional care and lowering the level of ICU transfer anxiety.

\section{Acknowledgements}

The authors gratefully acknowledge the Graduate School and Faculty of Nursing, Prince of Songkla University, Thailand. We are thankful to the research committee of the cardiac center in Nepal. We also express our gratitude to the participants of the study.

\section{Funding}

The scholarship awarded from Thailand's Education Hub for Southern Region of ASEAN Countries (TEH-AC) in Prince of Songkla University partly funded the data acquisition process.

\section{Author's Contributions}

JR conducted the entire study under the supervision of WS and LK. JR prepared the manuscript, which was critically reviewed and revised by WS and LK.

\section{Conflicts of Interest}

The authors declare no conflicts of interest regarding the publication of this paper.

\section{References}

[1] Manookian, A., Dehghan-Nayeri, N., Negarandeh, R. and Shali, M. (2015) The Lived Experiences of Intensive Care Patients on Transfer to a General Ward. Nursing Practice Today, 2, 52-61.

[2] Herbst, L.A., Desai, S., Bencoster, D., Jerardi, K., Meier, K.A., Statile, A.M. and 
White, C.M. (2018) Going Back to the Ward-Transitioning Care Back to the Ward Team. Transitional Pediatrics, 7, 314-325. https://doi.org/10.21037/tp.2018.08.01

[3] Cypress, B.S. (2013) Transfer Out of Intensive Care: An Evidence-Based Literature Review. Dimensions of Critical Care Nursing, 32, 244-261. https://doi.org/10.1097/DCC.0b013e3182a07646

[4] Cognet, S. and Coyer, F. (2014) Discharge Practices for the Intensive Care Patient: A Qualitative Exploration in General Ward Setting. Intensive and Critical Care Nursing, 30, 292-300. https://doi.org/10.1016/j.iccn.2014.04.004

[5] Cullaine, J.P. and Plowright, C.I. (2013) Patients and Relatives' Experiences of Transfer from the Intensive Care Unit to the Wards. Nursing in Critical Care, 18, 289-296.

[6] Herdman, T.H. and Kamitsuru, S. (2014) NANDA International Nursing Diagnoses: Definitions and Classification, 2015-2017. Wiley Blackwell, Oxford.

[7] Momennasab, M., Ghahramani, T., Yektatalab. S. and Zand, F. (2016) Physical and Mental Health of Patients Immediately after Discharge from Intensive Care Unit and 24 Hours Later. Trauma Monthly, 21, e29231.

[8] Zakerimoghadam, M., Ghyasvandian, S., Asayesh, M. and Jodaki, K. (2016) Prevalence of Anxiety in the Process of Transferring Patients from Cardiac Surgical Intensive Care Unit to the General Ward. Critical Care Nursing, 9, e8001.

[9] Kiliç, H.S. and Taştan, S. (2017) Cardiovascular Surgery Patients' Intensive Care Experiences and Transfer Anxiety. Gulhane Medical Journal, 59, 67-72.

[10] Ramsay, P., Huby, G., Thompson, A. and Walsh, T. (2014) Intensive Care Survivors' Experience of Ward-Based Care: Meleis' Theory of Nursing Transitions and Role Development among Critical Care Outreach Services. Journal of Clinical Nursing, 23, 605-615.

[11] Hosein, F.S., Bobrovitz, N., Berthelot, S., Zygun, D., Ghali, W.A. and Stelfox, H.T. (2013) A Systematic Review of Tools for Predicting Adverse Events Following Patient Discharge from Intensive Care Units. Critical Care, 17, Article No. R102. https://doi.org/10.1186/cc12747

[12] Lazarus, R.S. and Folkman, S. (1984) Stress, Appraisal and Coping. Springer Publishing Company, New York.

[13] Tung, H., Hunter, A. and Wei, J. (2008) Coping, Anxiety and Quality of Life after Coronary Artery Bypass Graft Surgery. Journal of Advanced Nursing, 61, 651-663.

[14] Chauvet-Gelinier, J.C. and Bonin, B. (2017) Stress, Anxiety and Depression in Heart Disease Patients: A Major Challenge for Cardiac Rehabilitation. Annals of Physical and Rehabilitation Medicine, 60, 6-12. https://doi.org/10.1016/j.rehab.2016.09.002

[15] Jamsahar, M., Navab, E., Yekaninejad, M.S. and Navidhamidi, M. (2018) The Effect of Provision of Information on Serum Cortisol in Patients Transferred from Coronary Care Unit to General Ward: A Randomised Controlled Trial. Intensive and Critical Care Nursing, 46, 38-43. https://doi.org/10.1016/j.iccn.2018.02.005

[16] Jodaki, K., Ghyasvandian, S., Kazemnejad, A. and Zakerimoghadam, M. (2017) Effect of Liaison Nurse Service on Transfer Anxiety of Patients Transferred from the Cardiac Surgery Intensive Care Unit to the General Ward. Nursing and Midwifery Studies, 6, e33478.

[17] Polit, D.F. and Beck, C.T. (2017) Nursing Research: Generating and Assessing Evidence for Nursing Practice. 10th Edition, Wolters Kluwer Health, Philadelphia

[18] Giammanco, M.D. and Gitto, L. (2016) Coping, Uncertainty and Health Related Quality of Life as Determinants of Anxiety and Depression on a Sample of Hospita- 
lized Cardiac Patients in Southern Italy. Quality of Life Research, 25, 2941-2956. https://doi.org/10.1007/s11136-016-1323-5

[19] Mishel, M.H. (1981) The Measurement of Uncertainty in Illness. Nursing Research, 30, 258-263. https://doi.org/10.1097/00006199-198109000-00002

[20] Carver, C.S. (1997) You Want to Measure Coping but Your Protocols' Too Long: Consider the Brief COPE. International Journal of Behavioral Medicine, 4, Article No. 92. https://doi.org/10.1207/s15327558ijbm0401_6

[21] Cooper, C., Katona, C., Orrell, M. and Livingston, G. (2006) Coping Strategies and Anxiety in Caregivers of People with Alzheimer's Disease: The LASER-AD Study. Journal of Affective Disorders, 90, 15-20. https://doi.org/10.1016/j.jad.2005.08.017

[22] Spielberger, C.D. (1983) Manual for State-Trait Anxiety Inventory (Form Y): Self Evaluation Questionnaire. Consulting Psychologists Press, Palo Alto.

[23] Häggström, M., Asplund, K. and Kristiansen, L. (2012) How Can Nurses Facilitate Patient's Transitions from Intensive Care? A Grounded Theory of Nursing. Intensive and Critcal Care Nursing, 28, 224-233. https://doi.org/10.1016/j.iccn.2012.01.002

[24] Forsberg, A., Lindgren, E. and Engström, A. (2011) Being Transferred from an Intensive Care Unit to Ward: Searching for the Known in the Unknown. International Journal of Nursing Practice, 17, 110-116.

[25] Salmond, S.W., Evans, B., Hamdi, H.A. and Saimbert, M.K. (2011) A Systematic Review of Relocation Stress Following In-House Transfer out of Critical/Intensive Care Units. JBI Library of Systematic Reviews, 9, 2684-2777. https://doi.org/10.11124/jbisrir-2011-76

[26] Joshi, A.S. (2015) A Novel Approach to Providing Nursing Care in Hospital of Nepal. Doctoral Dissertation, Okayama Prefectural University, Okayama.

[27] Aryal, R.P. (2018) Spirituality and Identity Reconstruction in Nepal: Living with HIV in Kathmandu and Pokhara Valleys. QUEST: Studies on Religion and Culture in Asia, 3, 1-14.

[28] Modica, M., Castiglioni, P., Minotti, A., Faini, A., Racca, V. and Ferratini, M. (2018) Psychological Profile in Coronary Artery By-Pass Patients vs Valve Replacement Patients Entering Cardiac Rehabilitation after Surgery. Scientific Reports, 8, Article No. 14381. https://doi.org/10.1038/s41598-018-32696-5 


\section{Appendix}

\section{State Anxiety Inventory (SAI)}

This questionnaire helps us identify your anxiety during transfer from ICU to general ward. Please mark " $x$ " in the box for each item that indicates your agreement with the statements in relation to your feeling of transfer from ICU to general ward, from when you were informed to be transferred to general ward till now. There are no wrong answers.

\begin{tabular}{|c|c|c|c|c|c|}
\hline S. No & Statements & $\begin{array}{c}\text { Not at all (1) } \\
(0 \%)\end{array}$ & $\begin{array}{c}\text { Somewhat (2) } \\
(1 \%-34 \%)\end{array}$ & $\begin{array}{c}\text { Moderately so (3) } \\
(35 \%-69 \%)\end{array}$ & $\begin{array}{c}\text { Very much so (4) } \\
(70 \%-100 \%)\end{array}$ \\
\hline 1. & I feel calm & & & & \\
\hline 2. & I feel secure. & & & & \\
\hline 3. & I am tense. & & & & \\
\hline 4. & I feel strained. & & & & \\
\hline 5. & I feel at ease. & & & & \\
\hline 6. & I feel upset. & & & & \\
\hline 7. & I am presently worrying over possible misfortunes. & & & & \\
\hline 8. & I feel satisfied. & & & & \\
\hline 9. & I feel frightened. & & & & \\
\hline 10. & I feel comfortable. & & & & \\
\hline 11. & I feel self-confident. & & & & \\
\hline 12. & I feel nervous. & & & & \\
\hline 13. & I am jittery. & & & & \\
\hline 14. & I feel indecisive. & & & & \\
\hline 15. & I am relaxed. & & & & \\
\hline 16. & I feel content. & & & & \\
\hline 17. & I am worried. & & & & \\
\hline 18. & I feel confused. & & & & \\
\hline 19. & I feel steady. & & & & \\
\hline 20. & I feel pleasant. & & & & \\
\hline
\end{tabular}

\section{Modified Mishel's Uncertainty in Illness Scale}

This questionnaire helps us identify your uncertainty in illness. These are the conditions you developed while being admitted to ICU till now in general ward. Please circle the response for each item that indicates your agreement with the statements. There are no wrong answers.

1. I do not know what is wrong with me.

Strongly agree (5) $\quad$ Agree (4) Undecided (3) Disagree (2) $\quad$ Strongly disagree (1)

2. I have a lot of questions without answers.

Strongly agree (5) $\quad$ Agree (4) Undecided (3) Disagree (2) $\quad$ Strongly disagree (1)

3. I am unsure if my condition is getting better or worse.
Strongly agree (5)
Agree (4) Undecided (3)
Disagree (2)
Strongly disagree (1) 
4. It is unclear how bad my symptoms after open heart surgery will be.
Strongly agree (5)
Agree (4) Undecided (3)
Disagree (2)
Strongly disagree (1)

5. The explanation health care providers give about my condition seem hazy to me.
Strongly agree (5)
Agree (4)
Undecided (3)
Disagree (2)
Strongly disagree (1)

6. The purpose of each treatment is clear to me.

Strongly agree (5) Agree (4) Undecided (3) Disagree (2) Strongly disagree (1)

7. When I have symptoms, I know what this means about my condition.
Strongly agree (5)
Agree (4)
Undecided (3)
Disagree (2)
Strongly disagree (1)

8. I do not know when to expect things will be done to me.

Strongly agree (5) $\quad$ Agree (4) Undecided (3) Disagree (2) $\quad$ Strongly disagree (1)

9. My symptoms continue to change unpredictably.

Strongly agree (5) $\quad$ Agree (4) Undecided (3) Disagree (2) $\quad$ Strongly disagree (1)

10. I understand everything explained to me.
Strongly agree (5)
Agree (4)
Undecided (3)
Disagree (2)
Strongly disagree (1)

11. The health care providers say things to me that could have many meanings.
Strongly agree (5)
Agree (4)
Undecided (3)
Disagree (2)
Strongly disagree (1)

12. I can predict how long my symptoms will last.
Strongly agree (5)
Agree (4)
Undecided (3)
Disagree (2)
Strongly disagree (1)

13. My treatment is too complex to figure out.
Strongly agree (5)
Agree (4)
Undecided (3)
Disagree (2)
Strongly disagree (1)

14. It is difficult to know if the treatments or medications I am getting are helping.
Strongly agree (5)
Agree (4)
Undecided (3)
Disagree (2)
Strongly disagree (1)

15. There are so many different types of staff; it's unclear who is responsible for what.
Strongly agree (5)
Agree (4)
Undecided (3)
Disagree (2)
Strongly disagree (1)

16. Because of the unpredictability of my symptoms, I cannot plan for the future.
Strongly agree (5)
Agree (4)
Undecided (3)
Disagree (2)

Strongly disagree (1)

17. The course of my symptoms keeps changing. I have good and bad days.
Strongly agree (5)
Agree (4)
Undecided (3)
Disagree (2)

Strongly disagree (1)

18. It's vague to me how I will manage my care after I have left the ICU.
Strongly agree (5)
Agree (4)
Undecided (3)
Disagree (2)

Strongly disagree (1)

19. I have been given many differing opinions about what is wrong with me.
Strongly agree (5)
Agree (4)
Undecided (3)
Disagree (2)

Strongly disagree (1)

20. It is not clear what is going to happen to me.
Strongly agree (5)
Agree (4)
Undecided (3)
Disagree (2)
Strongly disagree (1)

21. I usually know if I am going to have a good or bad day.
Strongly agree (5)
Agree (4) Undecided (3)
Disagree (2)
Strongly disagree (1)

22. The results of my test are inconsistent.
Strongly agree (5)
Agree (4)
Undecided (3)
Disagree (2)
Strongly disagree (1)

23. The effectiveness of my treatment is undetermined.
Strongly agree (5)
Agree (4)
Undecided (3)
Disagree (2)

Strongly disagree (1)

24. It is difficult to determine how long it will be before I can care for myself.
Strongly agree (5)
Agree (4)
Undecided (3)
Disagree (2)
Strongly disagree (1)

25 . I can generally predict the course of my illness.
Strongly agree (5)
Agree (4)
Undecided (3)
Disagree (2)
Strongly disagree (1) 
26. Because of the treatment, what I can do and cannot do keep changing.
Strongly agree (5)
Agree (4)
Undecided (3)
Disagree (2)
Strongly disagree (1)

27. I am certain they will not find anything else wrong with me.
Strongly agree (5)
Agree (4)
Undecided (3)
Disagree (2)
Strongly disagree (1)

28. The treatment I am receiving has a known probability of success.
Strongly agree (5)
Agree (4)
Undecided (3)
Disagree (2)
Strongly disagree (1)

29. They have not clarified me about my outcomes after surgery.
Strongly agree (5)
Agree (4)
Undecided (3)
Disagree (2)
Strongly disagree (1)

30. My physical distress is predictable; I know when it is going to get better or worse.
Strongly agree (5)
Agree (4)
Undecided (3)
Disagree (2)
Strongly disagree (1)

31. I can depend on the health care providers to be there when I need them.
Strongly agree (5)
Agree (4)
Undecided (3)
Disagree (2)
Strongly disagree (1)

32. The seriousness of my illness has been determined.
Strongly agree (5)
Agree (4) Undecided (3)
Disagree (2)
Strongly disagree (1)

33. The doctors and nurses use everyday language so I can understand what they are saying.
Strongly agree (5)
Agree (4)
Undecided (3)
Disagree (2)
Strongly disagree (1)

\section{Modified Brief COPE Inventory}

This questionnaire let us determine your use of coping abilities regarding the issues of surgery and transfer from ICU to general ward from admission to ICU till date. Please mark " $\mathrm{x}$ " in the box for each item that indicates your agreement with the statements. There are no wrong answers.

$1=\mathrm{I}$ have not been doing this at all $(0 \%)$.

$2=$ I have been doing this a little bit $(1 \%-34 \%)$.

$3=\mathrm{I}$ have been doing this a medium amount $(35 \%-69 \%)$.

$4=\mathrm{I}$ have been doing this a lot $(70 \%-100 \%)$.

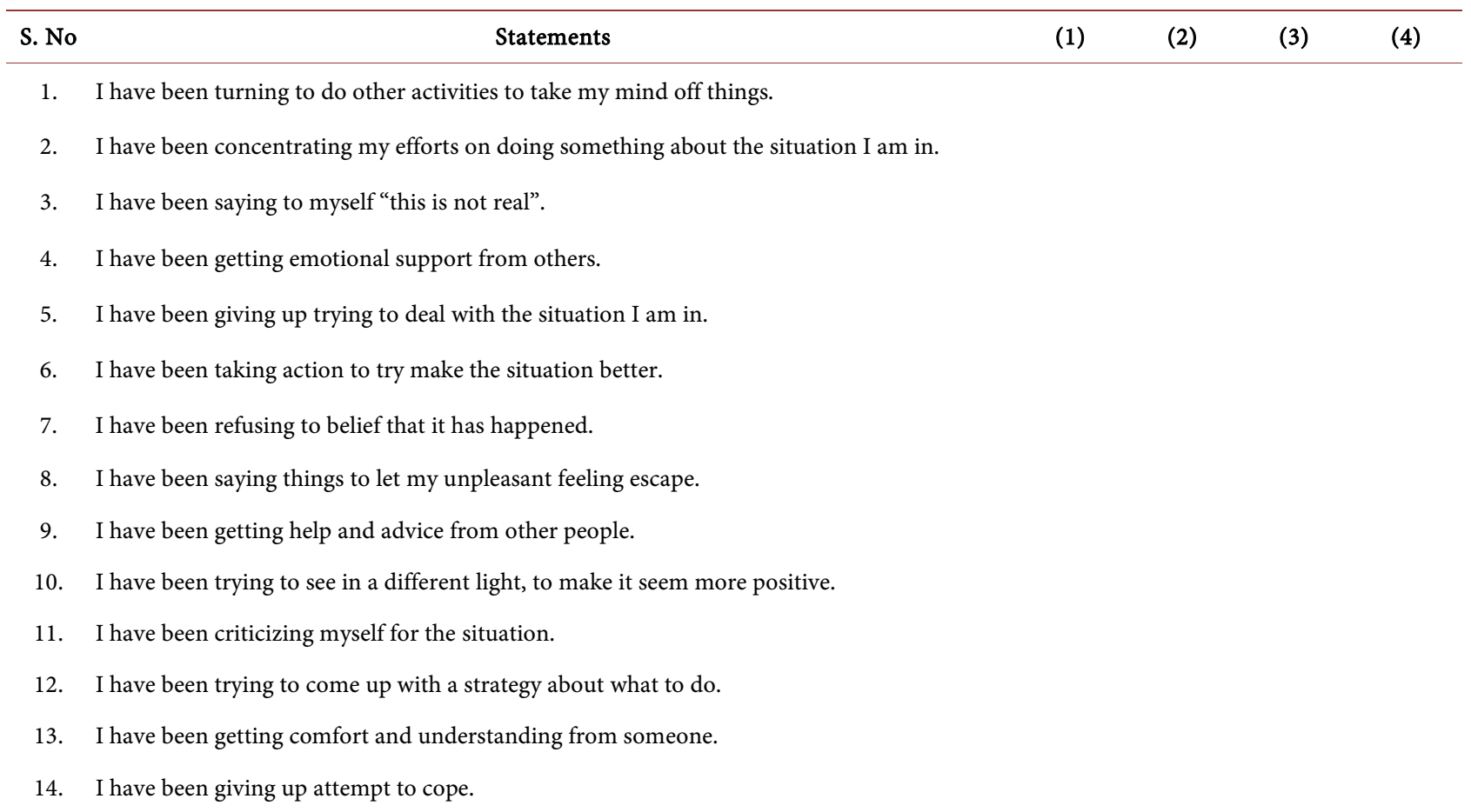




\section{Continued}

15. I have been looking something good in what is happening.

16. I have been making jokes about it.

7. I have been doing something to less think about it (such as listening to radio, reading, daydreaming, sleeping, talking to others).

18. I have been accepting the reality or of the fact that it has happened.

19. I have been expressing my negative feelings.

20. I have been trying to find comfort in my religion or spiritual belief.

21. I have been trying to get advice or help from other people about what to do.

22. I have been learning to live in general ward.

23. I have been thinking hard about what steps to take.

24. I have been blaming myself for thing that happened.

25. I have been praying or meditating.

26. I have been making fun of the situation I am in.

\section{Nurses' Support Questionnaire (NSQ)}

This questionnaire will make us understand about the support system you got from the nurses since your admission to ICU till current stay in general ward. How much are you satisfied with the statements given below? Choose best response indicating a sign of " $\mathrm{x}$ " in the box, that suite your interpretation of each statement. No answers are wrong.

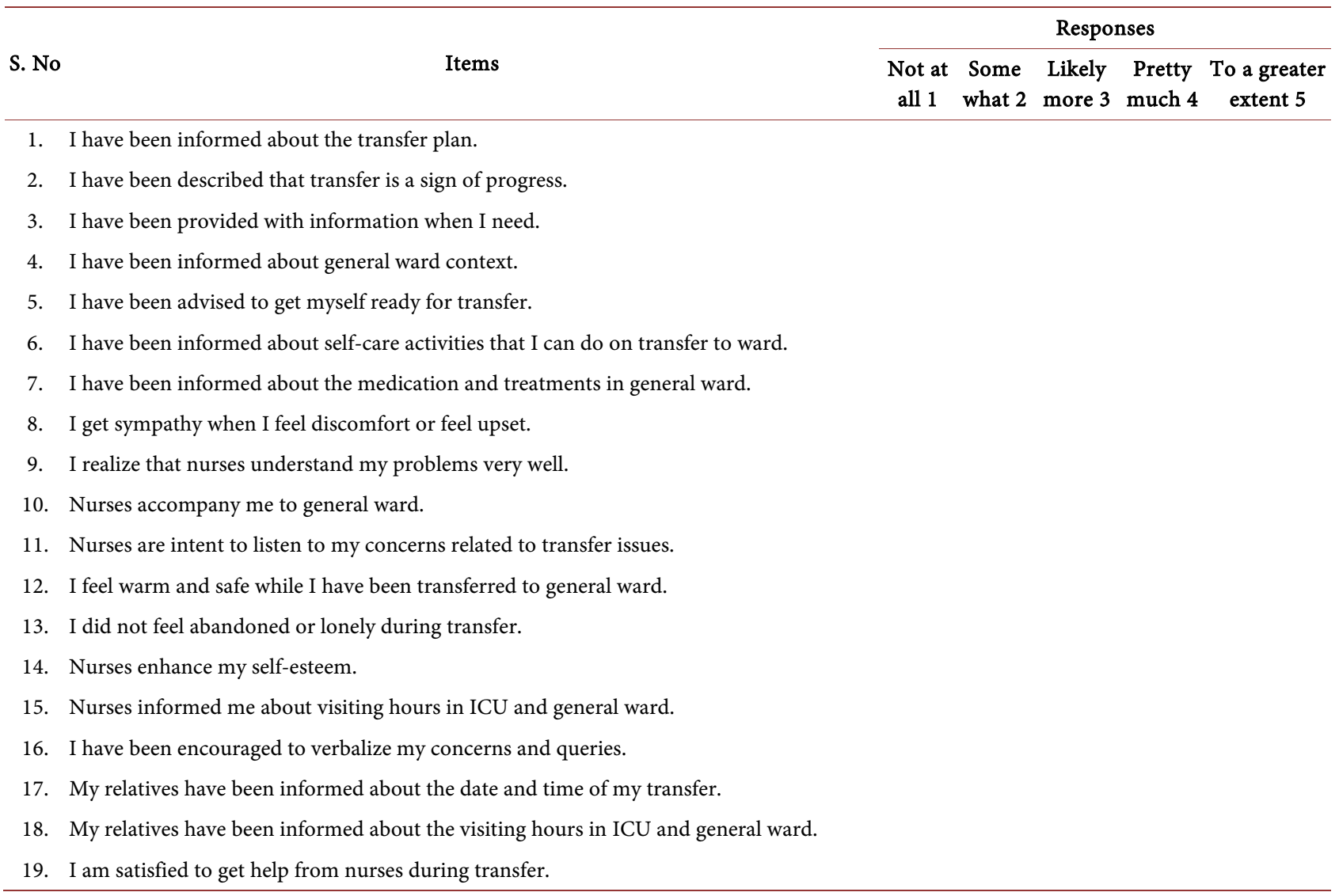

